\title{
PERSPECTIVE
}

\section{Bronchoalveolar lavage for the evaluation of interstitial lung disease: is it clinically} useful?

\author{
K.C. Meyer* and G. Raghu ${ }^{\#}$
}

ABSTRACT: Although the application of thoracic high-resolution computed tomography (HRCT) to clinical pulmonology has revolutionised the diagnostic approach to patients with suspected interstitial lung disease (ILD), additional testing is often needed to make a confident ILD diagnosis.

Bronchoalveolar lavage (BAL) can play a significant role in making an accurate and confident diagnosis of specific forms of ILD. When BAL is used in conjunction with comprehensive clinical information and HRCT, BAL nucleated immune cell patterns can frequently provide useful information for diagnostic evaluation and lessen the need to proceed to more invasive procedures, such as surgical lung biopsy.

Additionally, BAL can identify confounding conditions, such as infection or malignancy. However, BAL technique, and protocols for processing and analysing BAL fluid are critically important for providing useful information.

This perspective reviews the current status of using BAL as a diagnostic tool for the diagnosis of ILD.

KEYWORDS: Bronchoalveolar lavage, interstitial lung disease

B ronchoalveolar lavage (BAL) has gained widespread acceptance as a procedure that can be performed safely to retrieve respiratory secretions for the examination of cellular and acellular components for both diagnostic and research purposes [1-6]. When BAL was initially developed as a tool to sample respiratory secretions in animal models of lung disease and subsequently adapted as a clinical tool to study interstitial lung disease (ILD), it was perceived as holding considerable promise for the diagnosis and management of various forms of ILD, such as sarcoidosis, idiopathic pulmonary fibrosis (IPF) and hypersensitivity pneumonitis (HP). Indeed, hundreds of articles have been published in the medical literature over the ensuing decades as various centres around the world began to use BAL to identify agents of respiratory infections and examine correlates of changes in the composition of the airspace milieu associated with the presence of noninfectious parenchymal lung diseases. BAL is now routinely used as a tool to diagnose respiratory infections, evaluate patients with acute respiratory failure or evidence of diffuse parenchymal lung diseases, and monitor the status of transplanted lung allografts [7]. Despite the widespread use of BAL by pulmonologists, BAL cellular analysis, especially nucleated immune cell differential counts, may be underused in ILD diagnosis. Information derived from BAL appearance and differential cell counts can provide useful diagnostic clues if recognised and interpreted appropriately with an adequate awareness of the potential diagnoses that are associated with specific BAL cellular patterns.

In the early years following its introduction into clinical practice, bronchoscopy with BAL was perceived to hold great potential for diagnosis and management of ILD. It eventually became clear, however, that although BAL nucleated immune cell patterns often had characteristics that were highly consistent with various forms of ILD, such as sarcoidosis, BAL cell counts and differentials, lymphocyte subsets, or soluble components could not be relied upon to make a confident diagnosis for many specific forms of ILD if used as a standalone diagnostic test. As clinical pulmonary medicine evolved during the 1990s, high-resolution computed tomography (HRCT) of
AFFILIATIONS

*Division of Allergy, Pulmonary and Critical Care Medicine, Dept of Medicine, University of Wisconsin School of Medicine and Public Health, Madison, WI, and "Division of Pulmonary and Critical Care Medicine, University of Washington, Seattle, WA, USA.

CORRESPONDENCE

G. Raghu

Division of Pulmonary and Critical Care Medicine

University of Washington Campus Box 356166

Seattle

WA 98195-6522

USA

E-mail: graghu@u.washington.edu

Received:

April 292009

Accepted after revision:

April 102011

First published online:

May 032011

European Respiratory Journal Print ISSN 0903-1936 Online ISSN 1399-3003 
the chest came into widespread clinical use. As radiologists and clinicians became increasingly familiar with HRCT imaging patterns that were consistent with specific forms of ILD, the likelihood of making a confident diagnosis of specific ILD, such as IPF or sarcoidosis, is high when characteristic patterns are present and the technical quality of the HRCT is good $[8,9]$. Despite the increasing acceptance of obtaining a HRCT scan during the initial stages of evaluation of patients with ILD, many patients with new-onset ILD may not have the characteristic patterns that allow a diagnosis to be made with a high level of confidence by HRCT imaging alone. However, when clinical information and HRCT findings are combined with BAL fluid analysis and/or transbronchial lung biopsy, confident diagnoses may emerge that obviate the need for surgical lung biopsy. Although the BAL cell pattern can provide useful information that facilitates the establishment of a specific ILD diagnosis, this is only possible if the bronchoscopist uses an appropriate technique to obtain the fluid, and the differential cell count is performed according to good clinical laboratory practice by personnel with adequate experience in BAL cytological analysis and interpreted by an expert familiar with the diverse spectrum of specific forms of ILD. This article provides a perspective on the current and future use of BAL analysis in achieving a confident diagnosis of specific forms of ILD and the potential of BAL to assist in the management of various forms of ILD. Reaching a consensus on BAL technique and interpretive methods can bridge potential gaps that exist among pulmonologists worldwide in the application of BAL technique and analytic results to clinical practice.

\section{TECHNICAL ASPECTS OF PERFORMING BAL}

BAL technique is of paramount importance in obtaining a useful specimen $[10,11]$. To retrieve a specimen that is likely to reflect the types of inflammatory cells that are present in distal airspaces, a sufficient amount of isotonic saline should be instilled to adequately sample bronchoalveolar secretions from distal airspaces. The bronchoscopist should avoid contamination of the sample with proximal large airway secretions by maintaining the distal end of the bronchoscope in a wedged position in a segmental or subsegmental bronchus throughout the period of time required for the instillation and retrieval of saline aliquots. Additionally, dwell time should be minimised (aliquots should be aspirated immediately once the entire aliquot volume has been instilled). Many different BAL protocols have been published and consist of multiple aliquots that usually range from five or six aliquots of $20 \mathrm{~mL}$ each to four aliquots of $60 \mathrm{~mL}$ each. Some investigators consider the first aliquot to represent predominantly airway cells and secretions, and have recommended that this aliquot be kept separate and used for microbiological analysis, while subsequent sequential aliquots, usually three to four in number, should be pooled and used for cellular analysis because these are more representative of distal airspaces (alveolar sampling) [12]. Others combine all aliquots and do not differentiate between "bronchial" and "alveolar" sampling. There are no data that support one approach over the other, and many centres pool all retrieved BAL aliquots prior to submitting BAL for laboratory analysis. Additionally, there is no consensus on the optimal magnitude of negative pressure to retrieve BAL fluid once an aliquot has been instilled.
The right middle lobe (RML) and lingula of the left upper lobe are the areas of the lung that are more easily accessible and likely to allow good return of BAL fluid from patients when lavaged while in a supine position [11]. These areas have traditionally been used for lavage based on the perception that ILD is a "diffuse" parenchymal lung disease, as demonstrated on routine chest radiographs. Because patients with ILD are now routinely evaluated with HRCT images of the chest, HRCT images may be used to target areas of the lung that may yield cellular findings more reflective of the disease process, such as areas of ground glass attenuation, more prominent nodular profusion or fine reticulation. Since HRCT is sensitive in detecting patterns of otherwise occult segmental and/or patchy alveolar opacities or ground glass attenuation in patients with ILD, the HRCT images should be used as a guide to determine the site of BAL in order to include sampling from involved segments as indicated by the HRCT scan. However, no studies have been performed that demonstrate that the use of HRCT to target regions that show more prominent parenchymal change in certain geographic lung regions compared with traditionally used sites, such as the RML or lingula, improve the diagnostic yield of BAL. Ideally, the percentage of BAL fluid that is retrieved should be $\geqslant 30 \%$ for a patient with ILD. However, if ILD is superimposed on emphysema, airways may readily collapse with negative pressure and compromise fluid retrieval. Post-bronchoscopy fever (fever, chills and extreme malaise) may occur within hours of the BAL procedure as a consequence of proinflammatory mediator release, and these symptoms are more likely to occur if larger volumes of fluid are used for BAL [13].

\section{PROCESSING OF BAL FLUID FOR CELLULAR ANALYSES}

An accurate and statistically meaningful enumeration of the BAL nucleated immune cell profile requires examination of $\geqslant 300$ of these BAL cells randomly on a single slide, with accuracy further enhanced by analysing 400-500 cells (especially if more than one slide is analysed with the differential counts from each slide averaged). The relative numbers of bronchial and squamous epithelial cells should also be noted. Although many investigators have published BAL cell profiles for cohorts of healthy volunteers, laboratories that perform BAL analysis would ideally study a sizable group of normal volunteers and establish normal values in their own laboratories that correspond to their subject population and analytical methods. BAL slide preparations can be analysed in clinical haematopathology laboratories, but it is of key importance that personnel who analyse the BAL slide preparations and determine differential counts are adequately trained in the proper identification of BAL cells and in recognising various atypia that may be present on such preparations [11]. However, pulmonologists familiar with BAL cell patterns and ILD should interpret the BAL cell patterns and other findings.

BAL fluid obtained from healthy, never-smoking individuals should contain, on average, a majority of alveolar macrophages (AMs) (80-90\%), some lymphocytes (5-15\%), and very few neutrophils $(\leqslant 3 \%)$ or eosinophils $(<1 \%)$. While it is preferential to have laboratories establish normal values (including T-lymphocyte subsets), normal values reported by others may be used, especially if the BAL procedure that is used conforms to published techniques and values obtained for normal subjects. 
The presence of squamous epithelial cells suggests that the BAL fluid has been contaminated by oropharyngeal secretions, which may be a consequence of poor technique in performing the BAL or because the subject aspirated upper airway secretions. BAL cell differentials and total cell counts for ex-smokers should be similar to that of never-smokers. Smokers, however, generally have a significantly increased total BAL cell count, as well as total macrophages and neutrophils per microlitre of BAL fluid, but the BAL differential cell count for smokers appears to vary little from that for never-smokers or ex-smokers, except for a somewhat lower percentage of lymphocytes [5, 14]. Elderly subjects have been reported to have increases in lymphocytes and neutrophils in their differential cell counts, suggesting that advanced age may affect BAL cell differentials [15, 16]. Additionally, the total volume of retrieved fluid declines with advanced age due to the loss of elastic recoil and increased compliance of the aged lung. Some investigators have shown that total volumes of instilled saline that range from 100 to $250 \mathrm{~mL}$ appear to give similar cell differentials in individual patients with ILD $[17,18]$.

Infection can cause the subacute onset of diffuse lung infiltrates or coexist with noninfectious ILD. Therefore, BAL should be examined and screened for mycobacterial or fungal infection if clinical suspicion of an infectious aetiology warrants such testing. If bacterial infection is a possibility, an uncentrifuged BAL fluid specimen can be sent for quantitative bacterial culture. Uncentrifuged BAL fluid can also be sent for viral culture, although a centrifuged specimen may enhance virus detection via stains or viral nucleic acid probes. Centrifuged BAL specimens can also be used to culture Legionella, Mycoplasma and Chlamydia spp., mycobacteria and fungi, and cytocentrifuged specimens can be stained to detect the presence of intracellular bacteria, Pneumocystis jiroveci, mycobacteria or fungi [7].

\section{BAL CHARACTERISTICS AND THE DIAGNOSIS OF SPECIFIC TYPES OF ILD}

When performed with a standardised technique, expertly examined, and combined with clinical and imaging data, BAL differential cell counts and other characteristics can provide important information that contributes significantly to the diagnosis of specific ILDs (table 1) [1-3, 10, 19-22]. Additionally, cytopathological examination may be useful to detect malignancies (e.g. lymphangitic carcinoma and lymphomas) that can present as diffuse lung disease with a similar appearance to nonmalignant ILD on thoracic imaging [23]. Other observations, including the gross appearance of BAL fluid and identification of unusual cells (e.g. plasma cells, mast cells and foamy AMs), or foreign material can yield important information that may lead to a specific diagnosis. In the appropriate clinical setting, certain gross and cellular findings in BAL are highly suggestive or even virtually diagnostic of specific ILD entities (table 2), but these observations must be interpreted in the context of the patient's clinical presentation and radiological findings. Additionally, clinicians should recognise that radiographic imaging, including HRCT, may not appear particularly abnormal when certain forms of ILD (e.g. non-IPF interstitial pneumonias or HP) are present. If BAL is performed on a symptomatic patient who has radiographic imaging that is not particularly suspicious for the presence of ILD, an abnormal cell profile consistent with the presence of an "alveolitis" can indicate the need for additional investigation including lung tissue biopsy.

When BAL fluid is obtained and analysed from patients with ILD, differential cell counts obtained from slides prepared via cytocentrifugation usually show variations in the BAL cell differential that differ from patterns found in normal subjects. These patterns tend to reflect inflammatory cell profiles in affected lung tissues [1], but cell patterns may be obtained that are not typical for a specific ILD diagnosis, show little or no change from normal profiles or show mixed patterns with significant changes in the relative percentage of more than one type of nucleated immune cell [2]. Additionally, altered BAL cell profiles can be observed in various airway disorders (e.g. bronchiolitis, bronchitis, asthma, eosinophilic bronchitis, bronchiectasis and allergic bronchopulmonary aspergillosis). Nonetheless, certain cell patterns with significant changes in one or more BAL cell type can be highly consistent with or strongly suggest the presence of specific types of ILD. A recently published study of the predictive value of BAL for ILD diagnosis for a large cohort of patients $(n=3,118)$ suggested that BAL cell counts are most useful for relatively common ILD diagnoses, such as sarcoidosis, in contrast to relatively rare forms of ILD where BAL differential cell counts tend to have less diagnostic utility [24].

A BAL lymphocyte differential count $>25 \%$ is quite likely to be caused by ILD associated with granuloma formation (e.g. sarcoidosis and HP) or drug toxicity, if other possibilities, such as mycobacterial or fungal infection, are excluded. However, prominent BAL lymphocytosis can also be observed with other entities, such as cryptogenic organising pneumonia (COP), cellular nonspecific interstitial pneumonia (NSIP), lymphoid interstitial pneumonia (LIP) or non-LIP lymphoproliferative disorders. Extreme increases in BAL neutrophils are probably caused by infection, or relatively acute and diffuse lung injury. Eosinophil differential cell counts $\geqslant 25 \%$ are highly likely to be caused by eosinophilic lung disease, especially eosinophilic pneumonia (EP) if the presentation is acute [25, 26]. Increased numbers of mast cells have been associated with $\mathrm{HP}$, drug reactions, sarcoidosis, ILD associated with collagen vascular disease, IPF, COP, EP and malignancy. Plasma cells have been observed in BAL in $\mathrm{HP}$, drug reactions, EP, malignancy and infection [27]. AMs may also display certain morphological changes, such as: a foamy appearance in HP; markedly vacuolated cytoplasm with positive staining of vacuoles for fat in chronic aspiration pneumonitis; cytoplasmic inclusions associated with viral infection (e.g. cytomegalovirus pneumonia); ingested red blood cells (RBCs), and RBC fragments and haemosiderin with diffuse alveolar haemorrhage (DAH); ingested asbestos bodies; or other dust particles.

Grossly bloody lavage fluid, which may range from pink to red with acute haemorrhage, or to orange-brown if subacute, is characteristic of DAH, especially if sequentially retrieved BAL fluid aliquots do not show any decrease in the amount of bloody discoloration of the lavage fluid or (especially) if the discoloration increases [28]. If DAH is present, RBCs should be identifiable on the cytospin and AMs will stain positively for haemosiderin when stained with an iron stain if the onset of haemorrhage has preceded the time of BAL by $24-48 \mathrm{~h}$. Freshly retrieved BAL fluid that has a milky or light brown to whitish, cloudy appearance with flocculent debris that 


\section{TABLE 1 Bronchoalveolar lavage (BAL) findings that are useful in interstitial lung disease diagnosis}

BAL finding

Eosinophils $\geqslant 25 \%$

Lymphocytes $\geqslant 25 \%$

Neutrophils $\geqslant 50 \%$

Bloody fluid

High haemosiderin score

CD1a+ cells $>4 \%$

Milky BAL fluid with PAS-positive amorphous debris

Monotypic lymphocytes

Malignant cells

Squamous epithelial cells $>5 \%$

Bronchial epithelial cells $>5 \%$

Consistent interpretation/suggested diagnosis

Eosinophilic pneumonia

Sarcoidosis, HP, cellular NSIP, drug reaction, CBD, LIP, Iymphoproliferative disorder

AIP, DAD, AEIPF, pulmonary infection

Pulmonary haemorrhage, DAH

DAH, DAD

$\mathrm{PLCH}$

PAP

Pulmonary lymphomatous malignancy

Pulmonary malignancy

Unsuitable sample due to upper airway secretion contamination

BAL sample may be unsuitable for cell analysis

PAS: periodic acid-Schiff staining; HP: hypersensitivity pneumonitis; NSIP: nonspecific interstitial pneumonia; CBD: chronic beryllium disease; LIP: Iymphoid interstitial pneumonia; AIP: acute interstitial pneumonia; DAD: diffuse alveolar damage; AEIPF: acute exacerbation of interstitial pulmonary fibrosis; DAH: diffuse alveolar haemorrhage; PLCH: pulmonary Langerhans cell histiocytosis; PAP: pulmonary alveolar proteinosis.

settles out of the fluid to the bottom of its container prior to centrifugation suggests pulmonary alveolar proteinosis (PAP) as the likely diagnosis [29]. The diagnosis can be confirmed if the sediment, which is surfactant-derived lipoproteinaceous material, stains positively with periodic acid-Schiff staining.

Flow cytometric analyses of BAL cells have been reported by numerous investigators $[2,24,27]$ and alterations in BAL lymphocyte subsets have been examined extensively, especially for sarcoidosis [1, 2, 30-32]. The presence of a high CD4+/CD8+ T-lymphocyte ratio increases the likelihood of diagnosis of sarcoidosis when combined with BAL lymphocytosis, although the clinician must consider age as a factor in the elevated CD4+/ CD8+ ratio if the patient is elderly [16]. Nonetheless, it is debatable whether BAL lymphocyte subset data provides a clinical tool that contributes significantly to ILD diagnosis. The BAL CD4+ T-lymphocyte subset and CD4+/CD8+ T-lymphocyte ratio tend to be elevated in clinically active pulmonary sarcoidosis [31]. CD4+/CD8+ lymphocyte ratios in BAL from clinically healthy, younger adults usually range from 1.0 to 3.5, with average values between 1.5 and 2.0. An elevated CD4+/ CD8+ ratio of $\geqslant 3.5$ is relatively specific for sarcoidosis $[32,33]$. However, the sensitivity of an increased CD4+/CD8+ ratio is relatively low for sarcoidosis [32,33] and many patients do not have an elevated ratio or may even have a low ratio [34]. A depressed CD4+/CD8+ ratio has been observed in $\mathrm{HP}$, druginduced lung disease, COP, EP and IPF [27], but this ratio has not been shown to contribute meaningfully to the diagnosis of non-sarcoid ILD. Use of the CD4+/CD8+ ratio as a routine component of BAL analysis is questionable and may increase the cost of the procedure considerably.

Flow cytometry may be useful to support a diagnosis of pulmonary Langerhans cell histiocytosis (PLCH) if clinical data and imaging suggest this entity. PLCH is associated with increased ( $>4-5 \%$ of total cells) CD1a+ cells in BAL, although this may no longer be the case in later stages of disease $[35,36]$. However, macrophage autofluorescence may present insurmountable difficulties in obtaining reliable data and immunohistochemistry on prepared slides is more likely to provide reliable enumeration of the percentage of positively stained Langerhans cells [36]. Flow cytometry and/or immunocytochemistry can be useful in identifying monoclonal lymphocyte populations in order to support the diagnosis of a lymphoid malignancy, and should be obtained if clinical data suggest the possibility of pulmonary lymphoma and the clinical laboratory can perform appropriate analyses. Although flow cytometric analysis of BAL cells may provide useful information in certain situations, the routine use of flow cytometry to analyse BAL cell subpopulations to assist in the diagnosis of specific forms of ILD is of unclear benefit. However, flow cytometry of BAL cells can be obtained if it is likely to provide important diagnostic information.

One application of BAL that evolved over the past decade was the suggested incorporation of the BAL cellular findings into the major and minor criteria for the clinical diagnosis of IPF when surgical lung biopsy is not performed [37]. However, since the positive predictive value of the HRCT findings of the usual interstitial pneumonia (UIP) pattern for a diagnosis of IPF/UIP is $\geqslant 85 \%$ and a confident diagnosis of UIP by experienced radiologists can be made in $95 \%$ of cases with the characteristic pattern of UIP on HRCT images [38-41], invasive procedures including bronchoscopy, BAL and transbronchial lung biopsy may not be required to make a confident diagnosis of IPF in the appropriate clinical setting [9]. In fact, the features of the criteria for the UIP pattern has been precisely described in the new joint American Thoracic Society (ATS)/European Respiratory Society (ERS)/Japanese Respiratory Society (JRS)/Asociación Latinoamericana del Tórax (ALAT) statement; the new guideline states that the presence of the described UIP pattern on HRCT imaging is sufficient to make a diagnosis of IPF in the appropriate clinical setting and eliminates the need for BAL cellular analyses [42, 43]. However, an increased differential lymphocyte cell count in BAL from patients whose HRCT demonstrates a UIP pattern may raise the possibility of chronic $\mathrm{HP}$ or other diagnoses associated with BAL lymphocytosis. In a recent study, $8 \%$ of patients with a seemingly confident clinical and HRCT diagnosis of IPF had $>30 \%$ lymphocytes in BAL, an atypical finding for the diagnosis of UIP/IPF that suggested HP or another disorder as the actual diagnosis [44]. The new 


\section{TABLE 2 Bronchoalveolar lavage (BAL) findings: diagnostic value and disease correlations}

ILD suspected BAL helpful? Useful BAL findings Biagnostic value of BAL

\begin{tabular}{|c|c|c|c|}
\hline Sarcoidosis & Yes & $\uparrow \uparrow$ Lymph $\uparrow \uparrow$ CD4/CD8 T-cell ratio & $\begin{array}{l}\text { Highly consistent if CXR and/or HRCT shows typical changes } \\
\text { of BHL, nodules along bronchovascular structures }{ }^{\#}\end{array}$ \\
\hline HP & Yes & $\begin{array}{l}\uparrow \uparrow \text { Lymph } \\
\text { Plasma cells } \\
\text { Foamy macrophages }\end{array}$ & $\begin{array}{l}\text { Highly consistent if significant exposure to known } \\
\text { antigen combined with typical HRCT pattern } \\
\text { (ground-glass nodules in mid/upper lung regions) }^{\#}\end{array}$ \\
\hline Cellular NSIP & Yes & $\uparrow \uparrow$ Lymph & $\begin{array}{l}\text { Highly consistent if mid/lower lung region diffuse } \\
\text { GGO present }{ }^{\#}\end{array}$ \\
\hline Fibrotic NSIP & $+/-$ & $\begin{array}{l}\text { Variable } \\
\uparrow \text { Lymph } \\
\uparrow \text { Eos } \\
\uparrow \text { Neut }\end{array}$ & $\begin{array}{c}\text { Rule out infection/malignancy; absence of inconsistent } \\
\text { BAL findings plus typical/highly consistent HRCT } \\
\text { imaging is supportive of diagnosis }\end{array}$ \\
\hline Drug reaction & Yes & $\begin{array}{l}\uparrow \text { Lymph } \\
\uparrow \text { Eos }\end{array}$ & $\begin{array}{c}\text { Highly consistent if patchy/diffuse infiltrates plus drug } \\
\text { ingestion known to be pneumotoxic and cause } \\
\text { interstitial change }\end{array}$ \\
\hline AIP & Yes & $\begin{array}{c}\uparrow / \uparrow \uparrow \text { Neut } \\
\text { Exclude infection }\end{array}$ & Rule out infection/malignancy \\
\hline DAD & Yes & $\uparrow$ Neut reactive/desquamated Type II pneumocytes & $\begin{array}{l}\text { Type II pneumocyte changes are highly suggestive in } \\
\text { appropriate clinical setting with patchy/diffuse infiltrates }\end{array}$ \\
\hline Lymphangitic carcinoma & Yes & Malignant cells & Diagnostic \\
\hline Lymphoid malignancies & Yes & Monoclonal lymph & Diagnostic \\
\hline PLCH & Yes & $>4 \%$ CD1a+ cells & Highly consistent/diagnostic if typical HRCT changes present \\
\hline Lymphangioleiomyomatosis & $+/-$ & Exclude infection & $\begin{array}{c}\text { Rule out infection/malignancy; absence of inconsistent } \\
\text { BAL findings plus typical/highly consistent HRCT } \\
\text { imaging is supportive of diagnosis }\end{array}$ \\
\hline PAP & Yes & $\begin{array}{c}\text { Milky fluid } \\
\text { White/tan sediment PAS-positive sediment }\end{array}$ & Diagnostic if typical/consistent HRCT changes present \\
\hline Radiation pneumonitis & $+/-$ & $\uparrow$ Lymph & Highly consistent if thoracic irradiation occurred within window ${ }^{\#}$ \\
\hline EP & Yes & $\uparrow \uparrow$ Eos & $\begin{array}{l}\text { Eos }>25 \% \text { is diagnostic if correlated with typical/highly } \\
\text { consistent HRCT imaging (or typical/classic CXR pattern) }\end{array}$ \\
\hline COP & $+/-$ & $\begin{array}{c}\text { Variable alveolitis } \uparrow \text { Lymph } \\
\uparrow \text { Eos } \\
\uparrow \text { Neut }\end{array}$ & $\begin{array}{c}\text { Rule out infection/malignancy; evidence of inflammation } \\
\text { with typical/highly consistent HRCT imaging is supportive } \\
\text { of diagnosis }\end{array}$ \\
\hline Scleroderma & $+/-$ & $\begin{array}{c}\text { Variable } \\
\uparrow \text { Lymph } \\
\uparrow \text { Eos } \\
\uparrow \text { Neut }\end{array}$ & $\begin{array}{l}\text { Rule out infection/malignancy; evidence of inflammation } \\
\text { with typical/highly consistent HRCT imaging plus typical } \\
\text { clinical findings plus positive serological testing is } \\
\text { supportive of diagnosis }\end{array}$ \\
\hline Other CTD & $+/-$ & $\begin{array}{c}\text { Variable alveolitis } \uparrow \text { Lymph } \\
\uparrow \text { Eos } \\
\uparrow \text { Neut }\end{array}$ & $\begin{array}{l}\text { Rule out infection/malignancy; evidence of inflammation } \\
\text { with typical/highly consistent HRCT imaging plus typical } \\
\text { clinical findings plus positive serological testing is } \\
\text { supportive of diagnosis }\end{array}$ \\
\hline CBD & Yes & $\uparrow$ Lymph & $\begin{array}{l}\text { Rule out infection/malignancy; BAL Be-LPT plus } \\
\text { typical/highly consistent HRCT imaging is highly } \\
\text { consistent with diagnosis }\end{array}$ \\
\hline Asbestosis & $+/-$ & $\begin{array}{l}\text { Variable alveolitis } \\
\text { Asbestos fibres }\end{array}$ & $\begin{array}{c}\text { Rule out infection/malignancy; absence of inconsistent } \\
\text { BAL findings plus typical/highly consistent HRCT imaging } \\
\text { is supportive of diagnosis }\end{array}$ \\
\hline Silicosis & $+/-$ & $\uparrow$ AMs with $\uparrow$ silica particles & $\begin{array}{c}\text { Rule out infection/malignancy; absence of inconsistent } \\
\text { BAL findings plus typical/highly consistent HRCT imaging } \\
\text { is supportive of diagnosis }\end{array}$ \\
\hline $\begin{array}{l}\text { Chronic aspiration of } \\
\text { gastric secretions }\end{array}$ & Yes & $\begin{array}{l}\text { Lipid-laden AMs } \\
\text { Pepsin-like activity }\end{array}$ & $\begin{array}{l}\text { Rule out infection/malignancy; consistent BAL findings } \\
\text { plus typical/highly consistent HRCT imaging plus } \\
\text { consistent clinical history is supportive of diagnosis }\end{array}$ \\
\hline IPF & $+/-$ & $\begin{array}{l}\uparrow \text { Neut } \\
\uparrow \text { Eos }\end{array}$ & $\begin{array}{l}\text { Rule out infection/malignancy; absence of inconsistent } \\
\text { BAL findings plus typical/highly consistent HRCT } \\
\text { imaging is supportive of diagnosis; lymphocytosis }\end{array}$ \\
\hline DIP/RBILD & $+/-$ & Pigmented AMs $\pm \uparrow$ neut $\pm \uparrow$ eos & $\begin{array}{c}\text { suggests alternative diagnosis } \\
\text { Rule out infection/malignancy; presence of elevated } \\
\text { total smoker macrophage numbers plus absence of } \\
\text { inconsistent BAL findings plus typical/highly consistent } \\
\text { HRCT imaging is supportive of diagnosis }\end{array}$ \\
\hline LIP & $+/-$ & $\uparrow$ Lymph & $\begin{array}{l}\text { Rule out infection/malignancy; lymphocytosis plus } \\
\text { absence of inconsistent BAL findings plus typical/highly } \\
\text { consistent HRCT imaging is supportive of diagnosis }\end{array}$ \\
\hline DAH & Yes & $\begin{array}{l}\text { Bloody lavage } \\
\text { High Golde score }\end{array}$ & $\begin{array}{c}\text { Progressively bloody BAL fluid with sequential lavage } \\
\text { aliquots plus BAL macrophages with significant } \\
\text { amounts of haemosiderin are highly diagnostic but } \\
\text { do not establish specific cause }\end{array}$ \\
\hline Acute-onset ILD & $+/-$ & $\begin{array}{c}\uparrow \text { Lymph } \\
\uparrow \text { Eos } \\
\uparrow \text { Neut Infection/malignancy excluded } \\
\text { Infection excluded }\end{array}$ & $\begin{array}{l}\text { Marked lymphocytosis suggests HP or drug toxicity; } \\
\text { marked eosinophilia suggests acute EP as diagnosis; } \\
\text { reactive/desquamated type II pneumocytes suggests DAD } \\
\text { Rule out infection/malignancy; absence of inconsistent } \\
\text { BAL findings plus typical/highly consistent HRCT } \\
\text { imaging is supportive of diagnosis }\end{array}$ \\
\hline \multicolumn{4}{|c|}{$\begin{array}{l}\text { ILD: interstitial lung disease; HP: hypersensitivity pneumonitis; NSIP: nonspecific interstitial pneumonia; AIP: acute interstitial pneumonia; DAD: diffuse alveolar damage; PLCH: } \\
\text { pulmonary Langerhans cell histiocytosis; PAP: pulmonary alveolar proteinosis; EP: eosinophilic pneumonia; COP: cryptogenic organising pneumonia; CTD: connective tissue disease; } \\
\text { CBD: chronic beryllium disease; IPF: interstitial pulmonary fibrosis; DIP: desquamative interstitial pneumonia; RBILD: respiratory bronchiolitis-associated interstitial lung disease; LIP: } \\
\text { lymphoid interstitial pneumonia; DAH: diffuse alveolar haemorrhage; AEIPF: acute exacerbation of interstitial pulmonary fibrosis; Lymph: lymphocytes; Eos: eosinophils; Neut: } \\
\text { neutrophils; PAS: periodic acid-Schiff staining; AM: alveolar macrophage; CXR: chest radiography; HRCT: high-resolution computed tomography; BHL: bilateral hilar } \\
\text { lymphadenopathy; GGO: ground-glass opacity; Be-LPT: beryllium-induced lymphocyte proliferation test; +/-: while it is unlikely that BAL analyses will contribute to a specific } \\
\text { diagnosis, ruling out other entities such as infection, haemorrhage or malignancy by BAL analyses may be worthwhile in evaluating patients suspected to have these specific clinical } \\
\text { diagnoses; } \uparrow: \text { increased. \#: BAL cytological analysis and culture are useful to rule out infection and/or malignancy; }{ }^{\uparrow} \text { BAL lymphocyte subset determination is optional but is } \\
\text { particularly supportive if CD4/CD8 ratio } \geqslant 3.5 \text {. }\end{array}$} \\
\hline
\end{tabular}


evidence-based guideline for diagnosis and management of IPF remarks on this point and defers the decision for the need of BAL cellular analyses to individual experts with established BAL laboratories (that provide reliable cell differential counts) who may opt to use BAL cellular analyses in patients undergoing evaluation for the diagnosis of IPF at their centres to ensure the lack of findings that suggest a non-IPF form of ILD as the diagnosis [42].With the increasing use of HRCT to evaluate patients during the early stages of ILD, it is likely that patients with ILD will not manifest the precise pattern of UIP on HRCT. In this setting, many clinicians may routinely perform BAL for cellular analyses as a part of the initial evaluation of patients presenting with early- and new-onset ILD. Thus, the clinical utility of BAL cellular analyses as an useful intervention in narrowing the differential diagnoses of ILD in this setting is evident. It must, however, be recognised that for an accurate diagnosis, biopsy of the tissue with adequate sampling may still be required. The performance of transbronchial biopsy in the peripheral lesions of UIP, NSIP and desquamative interstitial pneumonia is not advised.

BAL can also assist in the diagnosis of acute-onset ILD (defined as a combination of illness $\leqslant 4$ weeks in duration, shortness of breath, hypoxaemia and diffuse radiographic infiltrates in a patient with no history of prior lung disease and no obvious risk factors for acute respiratory distress syndrome, such as sepsis or trauma). Diagnostic considerations in acute-onset ILD include infection and noninfectious ILD (acute interstitial pneumonia, acute $\mathrm{EP}, \mathrm{DAH}$, acute $\mathrm{HP}$, acute COP, drug toxicity or acute exacerbation of previously undiagnosed IPF). Examination of BAL fluid can detect infection or haemorrhage, and the BAL cell profile may show large numbers of eosinophils, which are strongly supportive of a diagnosis of acute EP. Additionally, large numbers of lymphocytes would suggest entities such as acute HP or drug toxicity, especially if accompanied by plasma cells and an appropriate exposure history. Bronchoscopy with BAL at the time of acute presentation may facilitate diagnosis and minimise procedural risk if a diagnosis can be made, and obviate the need to progress to a surgical lung biopsy.

\section{BAL AND THE MANAGEMENT OF ILD}

A role for BAL cellular analyses in the treatment of ILD (e.g. to initiate, direct and/or monitor pharmacological therapies) has not been established. One application of BAL to disease management, however, is its use to evaluate acute changes in symptoms and lung function. Several adverse events may occur in the course of interstitial diseases, including respiratory infection, drug reactions, haemorrhage or an acute exacerbation of the disease process, and BAL may help to identify the cause of clinical deterioration and facilitate timely intervention.

Many investigators have examined the role of BAL cellular profiles for monitoring disease activity and response to therapies in specific ILDs, and BAL cellular findings at the time of diagnosis have been reported by some investigators to reflect disease severity and predict the likelihood of disease progression. The degree of increase in BAL neutrophils has been correlated with disease severity and prognosis for both HP $[45,46]$ and IPF $[37,47-49]$, and BAL eosinophilia has been linked to more severe disease and worse prognosis in IPF [50, 51]. Similarly, the presence of increased neutrophils in BAL from patients with sarcoidosis has been associated with more progressive disease that is less likely to respond to immunosuppressive therapy [52]. BAL lymphocytosis has been linked to prognosis and response to therapy for some forms of idiopathic interstitial pneumonia (III). BAL findings from patients diagnosed with IPF when this diagnostic term did not necessarily exclude other IIPs, such as NSIP, suggested that higher BAL lymphocyte differential cell counts correlated with better prognosis and/or response to therapy [37, 48-50, 53]. A more recent study, although retrospective, demonstrated that BAL lymphocytosis in patients who appear to have fibrotic IIP suggests that the diagnosis is not IPF but rather NSIP, and BAL lymphocytosis was found to correlate with a better prognosis [54].

Lung lavage was initially used as a therapy for septic lung disease and PAP $[55,56]$. Although its therapeutic application is quite limited at this time, whole-lung lavage (WLL) is considered standard of care for PAP [57]. However, standardisation of technique and controlled clinical trials to determine the efficacy and durability of WLL as a therapy for PAP are lacking [57].

\section{FUTURE DIRECTIONS AND RESEARCH QUESTIONS}

BAL holds the potential to play an increasingly useful role in both the diagnosis and monitoring of patients with ILD, but many important issues have not been adequately resolved. These unresolved questions include the following. 1) What is the best technique for performing BAL (aliquot volume and number of aliquots; type and magnitude of negative pressure to retrieve instilled fluid; and pooling of aliquots for subsequent analysis)? 2) When and how should HRCT be used as a guide to determine the site of BAL in patients demonstrating diffusely distributed lesions in routine chest radiographs? 3) Should microbiological testing be performed whenever BAL is performed for cytological analysis to determine BAL cell total and differential cell count? 4) Should cytological analysis to detect malignant cells be performed whenever BAL is performed to evaluate diffuse lung disease? 5) Who should interpret BAL cellular preparations? 6) Can BAL combined with transbronchial lung biopsy synergistically increase diagnostic power and diminish the need for surgical lung biopsy? BAL may prove particularly helpful as treatments become available that may alter the profiles of BAL cells and/or noncellular components of lower respiratory tract bronchoalveolar surface liquid; if patients have favourable responses to such therapies, detection of these changes may support continued, ongoing treatment with specific agents. Additionally, multicentre studies that prospectively compare BAL with HRCT and histological patterns in large groups of patients will be facilitated by improvements in BAL standardisation [11]. Such studies have the potential to better define the underlying inflammatory and histological events associated with different HRCT appearances, which may reduce the need for biopsies and provide information that can guide effective therapies.

New tools that use genomic and proteomic characterisation of BAL cells and soluble components may eventually prove extremely useful in making an accurate ILD diagnosis, choosing and implementing potentially effective therapies, monitoring disease activity, and assessing the effect of therapeutic pharmacologic interventions. The recent development of DNA microarrays and other technologies that identify and monitor expression patterns of vast numbers of genes promises to provide important information about the pathobiology of various types of ILD $[58,59]$. Similarly, recent advances in proteomics that allow 
mapping and identification of multiple protein expression patterns by two-dimensional (2D) gel electrophoresis, image analysis, protein spot transfer and mass spectrometry now raise the real hope that the products of genes linked to disease pathogenesis can be identified and quantitated [60].

Determining gene and protein expression patterns over time, as reflected in BAL, can potentially identify the key molecules involved in the initiation and progression of the different ILDs, provide an accurate clinical diagnosis of specific ILDs without resorting to lung biopsy, and indicate targets for new and effective therapies. A global analysis approach has identified many genes that display increased expression in IPF [58]. Additionally, certain gene products that may play an important role in IPF pathogenesis, including pigment epitheliumderived factor [61], matrilysin [62] and osteopontin [63], have been identified via these methods and may serve as disease biomarkers. THONHOFER et al. [64] demonstrated that stimulated BAL cells from patients with sarcoidosis displayed up- or downregulation of $>1,000$ genes, including selective upregulation of $B-M Y B$, a potent growth factor for lymphocytes and regulator of apoptosis, and FABP4, a regulator of lipid metabolism and arachidonic acid uptake by macrophages. Not only can microarray genetic analysis provide characteristic gene expression patterns for specific ILD, but distinct expression patterns may differentiate one ILD from another. SELMAN et al. [65] recently validated the concept that gene expression patterns can be characteristic of specific ILDs by demonstrating that distinct gene expression patterns in lung tissue differed for patients with IPF versus those with HP.

A proteomics approach may also revolutionise the utility of BAL analysis for ILD diagnosis and management [60]. Characterising and quantitating proteins in biological specimens allow one to circumvent modifications of RNA that affect protein production and expression, and proteomic analytic techniques can be applied to acellular compartments, such as epithelial surface liquid retrieved via BAL or peripheral blood plasma. Early investigations that used electrophoretic techniques to examine protein profiles in BAL were able to show some differences between IPF and sarcoidosis [66, 67], and later studies using 2D electrophoretic techniques allowed enhanced fingerprinting of digested proteins from BAL supernatant fluids and demonstrated different profiles for IPF, sarcoidosis and HP $[68,69]$. More recently, ROTTOLI et al. [70] used 2D electrophoresis to construct protein maps from BAL fluid that were able to demonstrate differences between IPF, systemic sclerosis and sarcoidosis. The development of techniques that employ mass spectrometry combined with ionisation of peptides via matrix-assisted laser desorptionionisation or electrospray ionisation, or mass spectrometry combined with the use of surface chromatography to capture proteins on a chip surface (surface-enhanced laser desorptionionisation) coupled with greatly enhanced computational abilities and proteomic databases hold considerable promise for the study of ILD via this emerging technology, and these techniques have recently been applied to the study of markers in BAL fluid in patients with lung inflammation [71].

\section{SUMMARY AND CONCLUSIONS}

Although investigators have recognised the importance of the BAL procedure in clinical pulmonary medicine, unresolved questions concerning the BAL procedure and the clinical utility of BAL cellular analyses for the evaluation and management of ILD need to be addressed, and consensus reached among international experts. The technical aspects of performing BAL and analysing cellular features of BAL fluid need standardisation, and the status of the clinical utilisation of BAL findings in day-to-day clinical practice needs to be clarified so that principles of performing, analysing and interpreting BAL can be applied globally, avoiding wide variations in BAL technique and specimen analysis. BAL cellular patterns and other BAL findings can play an important role in the diagnosis of many forms of ILD, especially when these findings are obtained via a properly performed BAL procedure and analysis, and then combined with clinical data and HRCT imaging. In many instances, the combination of clinical information (history, examination and pulmonary function testing), HRCT imaging and BAL analysis can provide enough information to allow the clinician to be assured of a confident diagnosis and avoid more invasive testing to obtain lung tissue for histopathological diagnostic examination. BAL may be particularly beneficial for the fragile patient or patients at high risk for significant complications if subjected to surgical lung biopsy.

Universal adoption of a standardised BAL procedure will undoubtedly improve the chance of identifying clinically relevant differences between specific disorders and provide results that can be compared from centre to centre. A standardised procedure will enable centres to pool information to achieve the large patient groups necessary to more adequately investigate the value of BAL markers for the identification of specific disease entities, determining clinical disease activity, detecting disease progression, providing a prognosis and guiding treatment strategies. Identifying specific and sensitive markers that can address these problems remains an elusive but vitally important goal. Future application of BAL to the evaluation of ILD in concert with an evolving, improved understanding of the pathogenesis of specific ILDs, and the development of novel testing that uses genomic and proteomic characterisation of BAL fluids holds the potential to provide accurate diagnostic and prognostic information for patients with ILD and potentially play a role in choosing novel therapies for specific forms of ILD. Despite this significant diagnostic potential, very few patients have been investigated so far using gene and protein mapping approaches, and applications of the powerful techniques of gene and protein mapping to the study of ILD are still at a very early stage but have the potential to evolve rapidly in the coming years. Access to large collections of well-characterised clinical samples (BAL, lung biopsies and peripheral blood) to which these techniques can be applied, combined with HRCT or newer forms of thoracic imaging, will greatly facilitate our understanding of basic and clinical aspects of ILD pathobiology, and facilitate the identification of clinically useful biomarkers.

\section{STATEMENT OF INTEREST}

None declared.

\section{REFERENCES}

1 Costabel U, Guzman J. Bronchoalveolar lavage in interstitial lung disease. Curr Opin Pulm Med 2001; 7: 255-261. 
2 Costabel U, Guzman J. Bronchoalveolar lavage. In: Schwartz MI, King TE Jr, eds. Interstitial Lung Disease. Hamilton, BC Decker, 2003; pp. 114-133.

3 Drent M, Meyer KC, Baughman RP. Bronchoalveolar lavage. Prog Respir Res 2007; 36: 58-67.

4 Clinical guidelines and indications for bronchoalveolar lavage (BAL): report of the European Society of Pneumology Task Group on BAL. Eur Respir J 1990; 3: 937-976.

5 The BAL Co-operative Group Steering Committee. Bronchoalveolar lavage constituents in healthy individuals, idiopathic pulmonary fibrosis, and selected comparison groups. Am Rev Respir Dis 1990; 141: Suppl. 5, S169-S202.

6 Haslam PL, Baughmann RP, eds. Guidelines for the measurement of acellular components and recommendations for standardization of bronchoalveolar lavage (BAL). Eur Respir Rev 1999; 9: 25-157.

7 Meyer KC. Bronchoalveolar lavage as a diagnostic tool. Semin Respir Crit Care Med 2007; 28: 546-560.

8 Kanne JP. Interstitial lung disease (ILD): imaging finding, and the role of imaging in the evaluating the patient with known or suspected ILD. Semin Roentgenol 2010; 45: 3.

9 Schmidt SL, Sundaram B, Flaherty KR. Diagnosing fibrotic lung disease: when is high-resolution computed tomography sufficient to make a diagnosis of idiopathic pulmonary fibrosis? Respirology 2009; 14: 934-939.

10 Haslam PL, Baughman RP. Report of ERS Task Force: guidelines for measurement of acellular components and standardization of BAL. Eur Respir J 1999; 14: 245-249.

11 Baughman RP. Technical aspects of bronchoalveolar lavage: recommendations for a standard procedure. Semin Respir Crit Care Med 2007; 28: 475-485.

12 Martin TR, Raghu G, Maunder RJ, et al. The effects of chronic bronchitis and chronic air-flow obstruction on lung cell populations recovered by bronchoalveolar lavage. Am Rev Respir Dis 1985; 132: 254-260.

13 Dhillon DP, Haslam PL, Townsend PJ, et al. Bronchoalveolar lavage in patients with interstitial lung diseases: side effects and factors affecting fluid recovery. Eur J Respir Dis 1986; 68: 341-350.

14 Costabel U, Guzman J. Effect of smoking on bronchoalveolar lavage constituents. Eur Respir J 1992; 5: 776-779.

15 Meyer KC. Neutrophils and low-grade inflammation in the seemingly normal aging human lung. Mech Aging Develop 1998; 104: 169-181.

16 Meyer KC, Soergel P. Bronchoalveolar lymphocyte phenotypes change in the normal aging human lung. Thorax 1999; 54: 697-700.

17 Dohn MN, Baughman RP. Effect of changing instilled volume for bronchoalveolar lavage in patients with interstitial lung disease. Am Rev Respir Dis 1985; 132: 390-392.

18 Helmers RA, Dayton CS Floerchinger C, et al. Bronchoalveolar lavage in interstitial lung disease: effect of volume of fluid infused. J Appl Physiol 1989; 67: 1443-1446.

19 Baughman RP, Drent M. Role of bronchoalveolar lavage in interstitial lung disease. Clin Chest Med 2001; 22: 331-341.

20 Meyer KC. The role of bronchoalveolar lavage in interstitial lung disease. Clin Chest Med 2004; 25: 637-649.

21 Baughman RP. Is bronchoalveolar lavage clinically useful for everyday practice in interstitial lung disease? Pro: bronchoalveolar lavage. J Bronchology 1999; 6: 211-216.

22 Raghu G. Is bronchoalveolar lavage clinically useful for everyday practice in interstitial lung disease? Con: bronchoalveolar lavage. J Bronchology 1999; 6: 217-221.

23 Poletti V, Poletti G, Murer B, et al. Bronchoalveolar lavage in malignancy. Semin Respir Crit Care Med 2007; 28: 534-545.

24 Welker L, Jörres RA, Costabel U, et al. Predictive value of BAL cell differentials in the diagnosis of intnerstitial lung diseases. Eur Respir J 2004; 24: 1000-1006.

25 Allen JN, Davis WB. Eosinophilic lung diseases. Am J Respir Crit Care Med 1994; 150: 1423-1438.
26 Pope-Harman AL, Davis WB, Allen ED, et al. Acute eosinophilic pneumonia. A summary of 15 cases and review of the literature. Medicine (Baltimore) 1996; 75: 334-342.

27 Drent M, Jacobs JA. Bronchoalveolar lavage. In: Baughman RP, Du Bois RM, Lynch JP III, et al., eds. Diffuse Lung Disease: A Practical Approach. New York, Oxford University Press, 2004: pp. 56-64.

28 Fontenot AP, Schwarz MI. Diffuse alveolar hemorrhage. In: Schwartz MI, King TE Jr, eds. Interstitial Lung Disease. Hamilton, BC Decker, 2003: pp. 632-656.

29 Martin RJ, Coalson JJ, Rogers RM, et al. Pulmonary alveolar proteinosis: the diagnosis by segmental lavage. Am Rev Respir Dis 1980; 121: 819-825.

30 Drent M, Mansour K, Linssen C. Bronchoalveolar lavage in sarcoidosis. Semin Respir Crit Care Med 2007; 28: 486-495.

31 Hunninghake GW, Crystal RG. Pulmonary sarcoidosis: a disorder mediated by excess helper T-lymphocyte activity at sites of disease activity. N Engl J Med 1981; 305: 429-432.

32 Costabel U, Zaiss AW, Guzman J. Sensitivity and specificity of BAL findings in sarcoidosis. Sarcoidosis 1992; 9: Suppl. 1, 211-214.

33 Winterbauer RH, Lammert J, Selland M, et al. Bronchoalveolar lavage cell populations in the diagnosis of sarcoidosis. Chest 1993; 104: 252-261.

34 Kantrow SP, Meyer KC, Kidd P, et al. The CD4/CD8 ratio in BAL fluid is highly variable in sarcoidosis. Eur Respir J 1997; 10: 27162721.

35 Auerswald U, Barth J, Magnussen H. Value of CD1 positive cells in bronchoalveolar lavage fluid for the diagnosis of pulmonary histiocytosis X. Lung 1990; 169: 305-309.

36 Costabel U, Guzman J, Bonella F, et al. Bronchoalveolar lavage in other interstitial lung diseases. Semin Respir Crit Care Med 2007; 28: 514-524.

37 American Thoracic Society. Idiopathic pulmonary fibrosis: diagnosis and treatment. International consensus statement. American Thoracic Society (ATS) and the European Respiratory Society (ERS). Am J Respir Crit Care Med 2000; 161: 646-664.

38 Hunninghake GW, Lynch DA, Galvin JR, et al. Radiologic findings are strongly associated with a pathologic diagnosis of usual interstitial pneumonia. Chest 2003; 124: 1215-1223.

39 Lynch DA, Godwin D Jr, Safrin S, et al. High-resolution computed tomography in idiopathic pulmonary fibrosis: diagnosis and prognosis. Am J Respir Crit Care Med 2005; 172: 488-493.

40 Swensen SJ, Aughenbaugh GL, Myers JL. Diffuse lung disease: diagnostic accuracy of CT in patients undergoing surgical biopsy of the lung. Radiology 1997; 205: 229-234.

41 Mathieson JR, Mayo JR, Staples CA, et al. Chronic diffuse infiltrative lung disease: comparison of diagnostic accuracy of CT and chest radiography. Radiology 1989; 171: 111-116.

42 Raghu G, Collard HR, Egan JJ, et al. An official ATS/ERS/JRS/ ALAT statement: idiopathic pulmonary fibrosis: evidence-based guidelines for diagnosis and management. Am J Respir Crit Care Med 2011; 183: 788-824.

43 Raghu G. Idiopathic pulmonary fibrosis: guidelines for diagnosis and clinical management have advanced from consensus-based in 2000 to evidence-based in 2011. Eur Respir J 2011; 37: 743-746.

44 Ohshimo S, Bonella F, Cui A, et al. Significance of bronchoalveolar lavage for the diagnosis of idiopathic pulmonary fibrosis. Am J Respir Crit Care Med 2009; 179: 1043-1047.

45 Haslam PL, Dewar A, Butchers P, et al. Mast cells, atypical lymphocytes, and neutrophils in bronchoalveolar lavage in extrinsic allergic alveoliltis. Comparison with other interstitial lung diseases. Am Rev Respir Dis 1987; 135: 35-47.

46 Drent $\mathrm{M}$, van Velzen-Blad $\mathrm{H}$, Diamant $\mathrm{M}$, et al. Bronchoalveolar lavage in extrinsic allergic alveolitis: effect of time elapsed since antigen exposure. Eur Respir J 1993; 6: 1276-1281.

47 Lynch JPD, Standiford TJ, Rolfe MW, et al. Neutrophilic alveolitis in idiopathic pulmonary fibrosis. The role of interleukin-8. Am Rev Respir Dis 1992; 145: 1433-1439. 
48 Haslam PL, Turton CW, Lukoszek A, et al. Bronchoalveolar lavage fluid cell counts in cryptogenic fibrosing alveolitis and their relation to therapy. Thorax 1980; 35: 328-339.

49 Rudd RM, Haslam PL, Turner-Warwick M. Cryptogenic fibrosing alveolitis. Relationships of pulmonary physiology and bronchoalveolar lavage to response to treatment and prognosis. Am Rev Respir Dis 1981; 124: 1-8.

50 Watters LC, Schwarz MI, Cherniack RM, et al. Idiopathic pulmonary fibrosis: pretreatment bronchoalveolar lavage cellular constituents and their relationships with lung histopathology and clinical response to therapy. Am Rev Respir Dis 1987; 135: 696-704.

51 Peterson WMW, Monick M, Hunniknghake GW. Prognostic role of eosinophils in pulmonary fibrosis. Chest 1987; 92: 51-56.

52 Drent M, Jacobs JA, de Vries J, et al. Does the cellular bronchoalveolar lavage fluid profile reflect the severity of sarcoidosis? Eur Respir J 1999; 13: 1338-1344.

53 Schwartz DA, Helmers RA, Galvin JR, et al. Determinants of survival in idiopathic pulmonary fibrosis. Am J Respir Crit Care Med 1994; 149: 450-454.

54 Ryu YJ, Chung MP, Han J, et al. Bronchoalveolar lavage in fibrotic idiopathic interstitial pneumonias. Respir Med 2007; 101: 655-660.

55 Kylstra JA, Rausch DC, Hall KD, et al. Volume-controlled lung lavage in the treatment of asthma, bronchiectasis, and mucoviscidosis. Am Rev Respir Dis 1971; 103: 651-665.

56 Ramirez J, Schultz RB, Dutton RE. Pulmonary alveolar proteinosis: a new technique and rationale for treatment. Arch Intern Med 1963; 112: 419-431.

57 Huizar I, Kavuru MS. Alveolar proteinosis syndrome: pathogenesis, diagnosis, and management. Curr Opin Pulm Med 2009; 15: 491-498.

58 Agostini C, Miorin M, Semenzato G. Gene expression profile analysis by DNA microarrays: a new approach to assess functional genomics in diseases. Sarcoidosis Vasc Diffuse Lung Dis 2002; 19: 5-9.

59 Kaminski N, Rosas IO. Gene expression profiling as a window into idiopathic pulmonary fibrosis pathogenesis: can we identify the right target genes? Proc Am Thorac Soc 2006; 3: 339-344.

60 Bowler RP, Ellison MC, Reisdorph N. Proteomics in pulmonary medicine. Chest 2006; 130: 567-574.
61 Cosgrove GP, Brown KK, Schiemann WP, et al. Pigment epithelium-derived factor in idiopathic pulmonary fibrosis: a role in aberrant angiogenesis. Am J Respir Crit Care Med 2004; 170: 242-251.

62 Zuo F, Kaminski N, Eugui E, et al. Gene expression analysis reveals matrilysin as a key regulator of pulmonary fibrosis in mice and humans. Proc Natl Acad Sci USA 2002; 99: 6292-6297.

63 Pardo A, Gibson K, Cisneros J, et al. Up-regulation and profibrotic role of osteopontin in human idiopathic pulmonary fibrosis. PloS Med 2005; 2: e251.

64 Thonhofer R, Maercker C, Popper HH. Expression of sarcoidosis related genes in lung lavage cells. Sarcoidosis Vasc Diffuse Lung Dis 2002; 19: 5-9.

65 Selman M, Pardo A, Barrera L, et al. Gene expression profiles distinguish idiopathic pulmonary fibrosis from hypersensitivity pneumonitis. Am J Respir Crit Care Med 2006; 173: 188-198.

66 Lenz AG, Meyer B, Costabel U, et al. Bronchoalveolar lavage fluid proteins in human lung disease: analysis by two-dimensional electrophoresis. Electrophoresis 1993; 14: 242-244.

67 Uebelhoer M, Bewig B, Oldigs M, et al. Protein profile in bronchoalveolar lavage fluid from patients with sarcoidosis and idiopathic pulmonary fibrosis as revealed by SDS-PAGE electrophoresis and western blot analysis. Scand J Clin Lab Invest 1993; 53: 617-623.

68 Wattiez R, Hermans C, Cruyt C, et al. Human bronchoalveolar lavage fluid protein two-dimensional database: study of interstitial lung diseases. Electrophoresis 2000; 21: 2703-2712.

69 Magi B, Bini L, Perari MG, et al. Bronchoalveolar lavage fluid protein composition in patients with sarcoidosis and idiopathic pulmonary fibrosis: a two-dimensional electrophoretic study. Electrophoresis 2002; 23: 3434-3444.

70 Rottoli P, Magi B, Perari MG, et al. Cytokine profile and proteome analysis in bronchoalveolar lavage of patients with sarcoidosis, pulmonary fibrosis associated with systemic sclerosis and idiopathic pulmonary fibrosis. Proteomics 2005; 5: 1423-1430.

71 de Torre C, Ying S, Munson PJ, et al. Proteomic analysis of inflammatory biomarkers in bronchoalveolar lavage. Proteomics 2006; 6: 3949-3957. 